\title{
¿Por qué creció la desigualdad en España durante la Gran Recesión?: una interpretación desde el marxismo
}

Francisco Javier Murillo Arroyo'

\section{Resumen}

Desde el estallido de la crisis los niveles de desigualdad en España se han intensificado a un ritmo superior al de otras economías. El método marxista permite comprender las causas que explican la desigualdad creciente: considerando que el origen de la crisis se fundamenta en el deterioro de las condiciones de rentabilidad se puede entender que la reacción adoptada para superarla haya pretendido aliviar la creciente presión sobre la ganancia. Para ello, se ha desplegado un ajuste salarial sustentado en el deterioro de las condiciones laborales y en la contención de las rentas salariales, que ha disparado la desigualdad.

Palabras clave

desigualdad, explotación, salario relativo, crisis.

Clasificación JEL:

B51, J30, FO2

\section{Resumo}

Desde a eclosão da crise, os níveis de desigualdade na Espanha intensificaram-se mais rapidamente do que em outras economias. O método marxista permite compreender as causas que explicam a crescente desigualdade: considerando que a origem da crise está na deterioração das condições de lucratividade, pode-se entender que a reação adotada para a superar tem buscado amenizar a pressão crescente sobre o lucro. Para tanto, foi implantado um reajuste salarial com base na deterioração das condições de trabalho e na contenção da renda salarial, o que desencadeou a desigualdade.

\section{Palavras chave}

Desigualdade; Exploração; Salário relativo; Crise.

Why did the inequality rise during the Great Recession?: an interpretation from marxism 


\section{Abstract}

Since the outbreak of the crisis, the levels of inequality in Spain have intensified at a faster rate than in other economies. The Marxist method allows us to understand the causes that explain the growing inequality: considering that the origin of the crisis is based on the deterioration of profitability conditions, it can be understood that the reaction adopted to overcome it has sought to alleviate the growing pressure on profit. For this, a wage adjustment has been deployed based on the deterioration of working conditions and the containment of wage income, which has triggered inequality.

\section{Keywords}

Inequality; Exploitation; Relative wages; Crisis.

Artigo recebido em junho 2020

Artigo aprovado em agosto de 2020

\section{Introducción}

Desde el estallido de la crisis la preocupación en la esfera política y académica por el crecimiento de la desigualdad ha adquirido un protagonismo creciente (ROSER y CRESPO, 2016; DE ARRIBA, 2014). En el caso español los niveles de desigualdad se han disparado, situándose así como uno de los países menos igualitarios de toda la UE. ¿Cuáles son las fuerzas que subyacen bajo esta dinámica? El objetivo de este trabajo es precisamente el de revelar las causas que la explican a partir de la aplicación del método marxista.

El materialismo dialéctico en el que se basa el análisis marxista no se contenta con describir con detalle el fenómeno de la creciente desigualdad, sino que permite detectar sus causas explicativas últimas. El marco teórico basado en la teoría laboral del valor (TLV) permite comprender el vínculo entre las contradicciones inherentes al proceso de acumulación y la creciente desigualdad.

A partir de la detección de las causas que laten bajo el estallido de la crisis es posible comprender la virulenta reacción emprendida por el capital: la profunda regresión salarial que se ha desplegado en los últi- 
mos años es el principal factor explicativo de la desigualdad. Si bien este escenario es común al conjunto de economías de la OCDE, en el caso español, por las peculiaridades que muestra en su patrón de acumulación, se ha presentado de manera acentuada (ROSER y CRESPO, 2016).

El artículo queda dividido en cuatro secciones. A esta introducción le sigue un apartado en el que se exponen los rudimentos teóricos del análisis marxista, que posibilitan la detección de los mecanismos desencadenantes de la crisis vinculados al desarrollo ordinario de la acumulación capitalista. En el siguiente apartado, se expone el proceso mediante el que se materializa el estallido de la crisis y la reacción del capital en el caso español. A continuación, se valora su impacto sobre los niveles de desigualdad, vertebrando el análisis sobre el conflicto entre capital y trabajo. Por último, se cierra con las principales conclusiones derivadas del trabajo, resaltando que bajo la intensificación de la desigualdad subyace la necesidad objetiva por parte del capital de explotar crecientemente a los asalariados.

\section{Dificultades de valorización, crisis y desigualdad creciente}

\subsection{Las crisis como un fenómeno inexorable a la acumulación capitalista}

Únicamente a partir de una caracterización rigurosa de la crisis es posible comprender cabalmente el carácter de la fase por la que atraviesa actualmente la economía mundial y el caso español en particular. A diferencia de otras explicaciones que consideran que la crisis obedece a una mala gestión de la acumulación capitalista, el análisis marxista considera que responde a la presión a la que, indefectiblemente, queda sometida la rentabilidad (MATEO, 2014). La TLV se erige como el eje vertebrador de este enfoque, del que emanan las principales categorías analíticas para comprender la dinámica de acumulación capitalista y, por supuesto, también la teoría sobre las crisis que de ella se deriva (ARRIZABALO, 2014). El método dialéctico 
en el que se basa trasciende la mera manifestación superficial de la realidad social, que no es evidente, y revela el contenido esencial de las relaciones sociales que la subyacen (GILL, 2002).

De ahí que ahonde en las causas últimas que cortocircuitan la acumulación, basando su explicación en las propias contradicciones que resultan inherentes a este proceso. Desde esta perspectiva, se considera que el desarrollo ordinario de la acumulación genera dificultades crecientes de valorización para el capital que, en un momento u otro, se acaban manifestando en la forma de crisis. Considerando que el principal factor determinante de la inversión es la rentabilidad, el enfoque basado en la TLV plantea que la presión que estructuralmente se cierne sobre la tasa de ganancia acaba interrumpiendo la acumulación.

Bajo esta tendencia se encuentra el incesante proceso de mecanización que caracteriza a la acumulación capitalista: a través de la competencia, la ley del valor impone la necesidad de mecanización creciente a cada uno de los capitales individuales que, en conjunto, integran al capital global. Por una parte, el capital está interesado en la mecanización del aparato productivo para favorecer la subsunción real de los asalariados, mediante la incorporación de medios de producción que sustituyan al trabajo vivo. Por otra, representa la principal alternativa para reducir los costes unitarios de producción, en el marco competitivo en el que se relacionan los capitales entre sí.

Pero la mecanización acaba generando funestas consecuencias para el proceso de acumulación: aunque, a nivel particular, permite a los capitales individuales mejorar su posición en el proceso de apropiación de beneficios, a nivel global deteriora la capacidad de generar valor nuevo del capital en su conjunto. El cambio técnico modifica la estructura de costes del capital, promoviendo un mayor peso relativo del capital constante en detrimento del variable (BOUNDI, 2014). Considerando que este último componente del capital es el responsable de la generación del plusvalor se puede entender cómo la mecanización mina la capacidad del capital global de crear valor nuevo. 
Entendiendo que la tasa de ganancia se define como la relación entre la masa total de plusvalor (pv), de la que se nutre el beneficio, y el capital total invertido, se puede afirmar que, como consecuencia de la menor capacidad de generación de valor nuevo que provoca la mecanización, el esfuerzo inversor que se debe hacer en forma de capital para conseguir el mismo volumen de beneficios es cada vez mayor. $\mathrm{O}$, dicho de otra manera, las condiciones de rentabilidad tienden a deteriorarse tendencialmente como consecuencia del funcionamiento ordinario del proceso de acumulación.

¿Cómo contrarrestar esta presión? Pueden aparecer diferentes fuerzas que permitan neutralizar, total o parcialmente, su efecto sobre la rentabilidad. Aunque existen otras vías para conseguirlo, la que el capital puede desplegar con más facilidad de manera sostenida en el tiempo es el incremento de la tasa de plusvalor (p'), definida como la relación entre pv y el capital variable (v). Es decir, mediante una modificación del reparto del valor nuevo generado favorable a los intereses del capital y en detrimento, obviamente, de los salarios. De este modo, la principal alternativa para neutralizar los efectos de la tendencia decreciente de la tasa de ganancia, se sitúa en el terreno de la distribución del ingreso nuevo creado en el proceso productivo. La insuficiente capacidad de generación de plusvalor por parte del capital se intenta paliar mediante un nuevo marco de distribución del ingreso favorable a los beneficios.

\subsection{Desigualdad creciente como resultado ordinario de la acumulación capitalista}

El análisis marxista considera que la desigualdad no solo es el resultado de la acumulación capitalista sino también su punto de partida. Para que la valorización del capital se ponga en marcha se requiere la existencia de dos clases sociales claramente diferenciadas: un grupo de sujetos que ostente la propiedad de los medios de producción, que conforma la clase capitalista, y otro compuesto por personas alejadas 
de ella, los asalariados. El funcionamiento ordinario del propio proceso de acumulación activa ciertos mecanismos que garantizan no solo la perpetuación de estas diferencias sino también su agudización.

La principal reacción del capital frente a la tendencia decreciente de la tasa de ganancia es el incremento de $p^{\prime}$ lo que implica una distribución del ingreso cada vez más favorable para los intereses del capital.

El ingreso no es más que la expresión monetaria del valor generado en el proceso productivo. El uso de la fuerza de trabajo del asalariado en este proceso propicia la generación de una determinada cantidad de valor nuevo $(v+p v)$ que será distribuido entre ambas clases sociales. El trabajador recibe la cantidad correspondiente al valor de su fuerza de trabajo $(v)$ mientras que el resto, el plusvalor ( $p v)$, es la fuente de la que se nutre la ganancia capitalista². En esto consiste el proceso de explotación: la clase capitalista se apropia de una fracción del valor nuevo generada por los trabajadores asalariados.

Las dificultades de valorización del capital radican en la pérdida de capacidad por parte del capital de generar valor nuevo, precisamente por el creciente protagonismo del componente constante frente al variable. De ahí que el capital reaccione promoviendo una distribución de este valor nuevo favorable a los beneficios: la relación entre la masa de plusvalor ( $p v)$ y el capital variable $(v)$, reflejada en $p$ ' tiende a crecer. $\mathrm{O}$, lo que es lo mismo, el grado de explotación de los trabajadores asalariados tiende a incrementarse.

El capital dispone de dos vías para conseguirlo. Por una parte, el incremento del plusvalor a través del método absoluto implica, fundamentalmente, una reducción del salario real o bien una extensión de la jornada de mayor intensidad que la mejora que, eventualmente, pudiera experimentar el salario real. Esta alternativa presenta ciertas restricciones ya que existen límites objetivos a la prolongación de la jornada y, además, se presenta de manera más evidente para los asalariados lo que aumenta las posibilidades de que desarrollen estrategias de oposición frente a ella. 
De ahí que sea más común el despliegue de la vía relativa para el incremento de la tasa de plusvalor. Esta estrategia se basa en alcanzar un avance de la productividad de mayor intensidad que el progreso que pueda experimentar el salario real. De esta manera, la porción del trabajo necesario durante la jornada laboral se reduce, incrementando así la masa de plusvalor generada. Y todo ello se consigue en un clima de mayor estabilidad porque es compatible con mejoras del salario real y, además, la división de la jornada laboral entre el trabajo necesario y el plustrabajo no se presenta de manera evidente a los trabajadores.

El incremento de la productividad que promueve la competencia capitalista posibilita un escenario de mejora del salario real, es decir, de la capacidad adquisitiva de los asalariados. Obviamente, este avance salarial no es automático, sino que su materialización depende de la forma en la que se articule el conflicto entre capital y trabajo, así como de los propios requerimientos de rentabilidad.

No obstante, la perspectiva relativa sobre la distribución del ingreso es mucho más precisa que la absoluta, ya que permite valorar la trayectoria de los salarios en función del grado de desarrollo económico alcanzado por la sociedad en cada momento. El salario relativo (SR), entendido como la participación de los salarios en el ingreso total, representa el elemento central de la teoría del salario de Marx (2.1).

$$
S R=v /(v+p v)=(v / v) /[(v+p v) / v]=1 /\left(1+p^{\prime}\right)
$$

Esta categoría permite poner de relieve el carácter antagónico que presentan salarios y beneficios, incluso en escenarios de mejora de la productividad al prevalecer la pugna distributiva por el progreso material alcanzado. El avance de la productividad genera una caída en el valor de la fuerza de trabajo al permitir la reproducción de la misma masa de valores de uso en un periodo de tiempo menor. Si, ante esta situación, el salario real se mantiene "aunque el nivel de vida absoluto del obrero seguiría siendo el mismo, su salario relativo, y por tanto su 
posición social relativa, comparada con la del capitalista, habrían bajado" (MARX, 1865: p. 53, énfasis en el original).

El SR está sometido a una presión estructural a la baja como consecuencia de las crecientes dificultades de valorización a las que se enfrenta la acumulación capitalista: su valor tiende a caer como resultado de la reacción que adopta el capital para neutralizar los efectos derivados de la caída tendencial de la tasa de ganancia.

Según se ha expuesto más arriba, el capital promoverá una distribución del ingreso favorable a sus intereses: al ver deteriorada su capacidad de generar valor requiere la apropiación de una porción creciente del valor nuevo creado en la forma de beneficios. Para ello, promueve un incremento de $p$ 'que provocará una caída en la participación de los salarios en el ingreso total.

Esta es la ley de depauperación relativa de los asalariados: su participación en la distribución del ingreso representa una fracción cada vez más reducida de la magnitud total. Al quedar definida como una tendencia de carácter estructural, se puede materializar con diferente intensidad, e incluso podría quedar contrarrestada, en función de diferentes elementos vinculados a la realidad social concreta. Y responde a los requerimientos de rentabilidad por parte del capital, que presenta una necesidad objetiva de explotar de manera cada vez más intensa a los asalariados.

\section{La singularidad de la crisis en España}

\subsection{Crisis y estructura productiva}

A partir del marco general definido en el apartado anterior se incorporan diferentes elementos de la realidad social para comprender la materialización del estallido de la crisis mundial de 2007 en el caso español.

Durante la fase que antecede a la crisis la acumulación en España había presentado un carácter más dinámico que el resto de las economías de la OCDE. No obstante, el patrón de crecimiento había comenzado a presentar síntomas de agotamiento antes de que se 
produjese la interrupción de la acumulación: entre los años 2002 y 2007 la tasa de ganancia sufre una caída del 4,8\% (cálculos propios a partir de series del INE para los beneficios y del IVIE para el capital) 3 . Las ratios sectoriales del Banco de España confirman también este deterioro: durante ese mismo periodo, el indicador que relaciona el resultado económico neto de las sociedades no financieras con el total de su activo cayó en un 15,7\%. Obviamente, a partir de ese año la caída de la rentabilidad se agudizó, alcanzando la caída una magnitud del 37,2\% hasta el año 2016.

Precisamente es a partir del año 2002, coincidiendo con la erosión de la rentabilidad, cuando germinan las burbujas especulativas, de especial intensidad en el mercado inmobiliario. En contra de otras interpretaciones que las sitúan como el principal factor explicativo de la crisis, este fenómeno se concibe como el reflejo de las crecientes dificultades de valorización a las que se enfrenta el capital. Como consecuencia de ellas, hay una ingente masa de capital que huye del ámbito productivo y busca refugio en la esfera especulativa, espoleada por la liberalización de la ley del suelo y el contexto de bajos tipos de interés. De ahí que la parte ficticia de la ganancia adquiera un protagonismo creciente durante este periodo. Al quedar basada en un proceso de valorización sin sustrato real, el estallido de la burbuja no hace sino revelar la insuficiencia del capital para generar plusvalía.

Durante una fase caracterizada por la desregulación financiera y los bajos tipos de interés se pretende avivar la acumulación mediante la expansión del crédito, lo que propicia un escenario de enorme inestabilidad en el que el segmento financiero del capital aparece hipertrofiado. Como consecuencia de las restricciones impuestas por el Pacto de Estabilidad y Crecimiento (PEC), el proceso de endeudamiento tuvo un carácter fundamentalmente privado. Y no solo fueron las familias las que se vieron obligadas a recurrir al crédito para sustentar su consumo ante la contención salarial sino también las empresas, que de este modo pretendían atenuar su menor capaci- 
dad de generar beneficios. Sin embargo, la expansión del crédito no resuelve las contradicciones inherentes a la acumulación capitalista, sino que únicamente posterga su manifestación, para que acaben materializándose posteriormente con mayor virulencia.

Por lo tanto, la esfera financiera no presenta una dinámica propia capaz de explicar por sí misma la crisis sino que queda estrechamente ligada a la del ámbito productivo, caracterizada por las crecientes dificultades para sostener la rentabilidad.

Según se ha desarrollado más arriba, la reacción del capital para abordar la crisis se asienta fundamentalmente sobre el incremento de $p^{\prime}$, aunque también la destrucción de capital contribuye a restaurar los niveles de ganancia. Todo ello con la pretensión de favorecer un escenario de distribución del producto más favorable a los beneficios, ante el deterioro de la capacidad del capital de generar valor nuevo. En el caso español, la estrategia se ha basado en la combinación de ambos métodos de extracción del plusvalor: el limitado avance que ha experimentado la productividad explica el hecho de que la vía relativa haya resultado insuficiente. Los indicadores de productividad únicamente muestran un avance significativo durante los años más duros de la crisis, como consecuencia del fuerte proceso de destrucción de empleo. A partir de entonces, se ha vuelto a la senda característica de las últimas décadas, mostrando un magro avance inferior al de las economías del entorno: durante el subperiodo 2014-2017, la tasa media de variación anual promedio de la productividad fue del 0,28\% frente al 0,73\% de la eurozona (cálculos propios a partir de datos de EU Klems).

La estructura productiva española, orientada hacia sectores poco permeables al cambio técnico, explica, en buena medida, el exiguo avance de la productividad. En el Cráfico 3.1 se muestran los Índices de Especialización (IE) de España respecto a la eurozona, que permiten constatar su específica estructura productiva. La ventaja de su utilización es que permite detectar, no solo las principales transformaciones 
en la estructura productiva española, sino si éstas han sido acordes con las experimentadas por las economías de referencia. Los IE relacionan el peso relativo de cierto sector (i), en producción o empleo, en dos economías diferentes ( $\mathrm{A}$ y $\mathrm{B}$ ) del siguiente modo:

$$
\mathrm{IE}_{i}^{A-B}=\left(\frac{\mu_{i}^{A}}{\mu_{i}^{B}}\right) \times 100
$$

Donde $\mu_{i}^{j}$ representa el peso relativo del sector i, en producción o empleo, en la economía j. De esta manera, un valor superior (inferior) a 100 del indicador IEi, ${ }^{A-B}$ representa una sobreespecialización (infraespecialización) de la economía A respecto a la $B$ en el sector i. Si se compara la estructura productiva española con la relativa al conjunto de la eurozona se ponen de manifiesto las peculiaridades mencionadas que han limitado el avance técnico y, por tanto, el desarrollo de la productividad ${ }^{4}$.

Tradicionalmente, la estructura económica española ha quedado asentada sobre ramas productivas poco susceptibles a la incorporación del cambio técnico, limitando así las posibilidades de mecanización y, por lo tanto, de avance de la productividad. Durante el reciente periodo recesivo no ha habido transformaciones significativas en la estructura económica, de tal manera que el sesgo mencionado en relación con el resto de economías de la eurozona se ha perpetuado. 


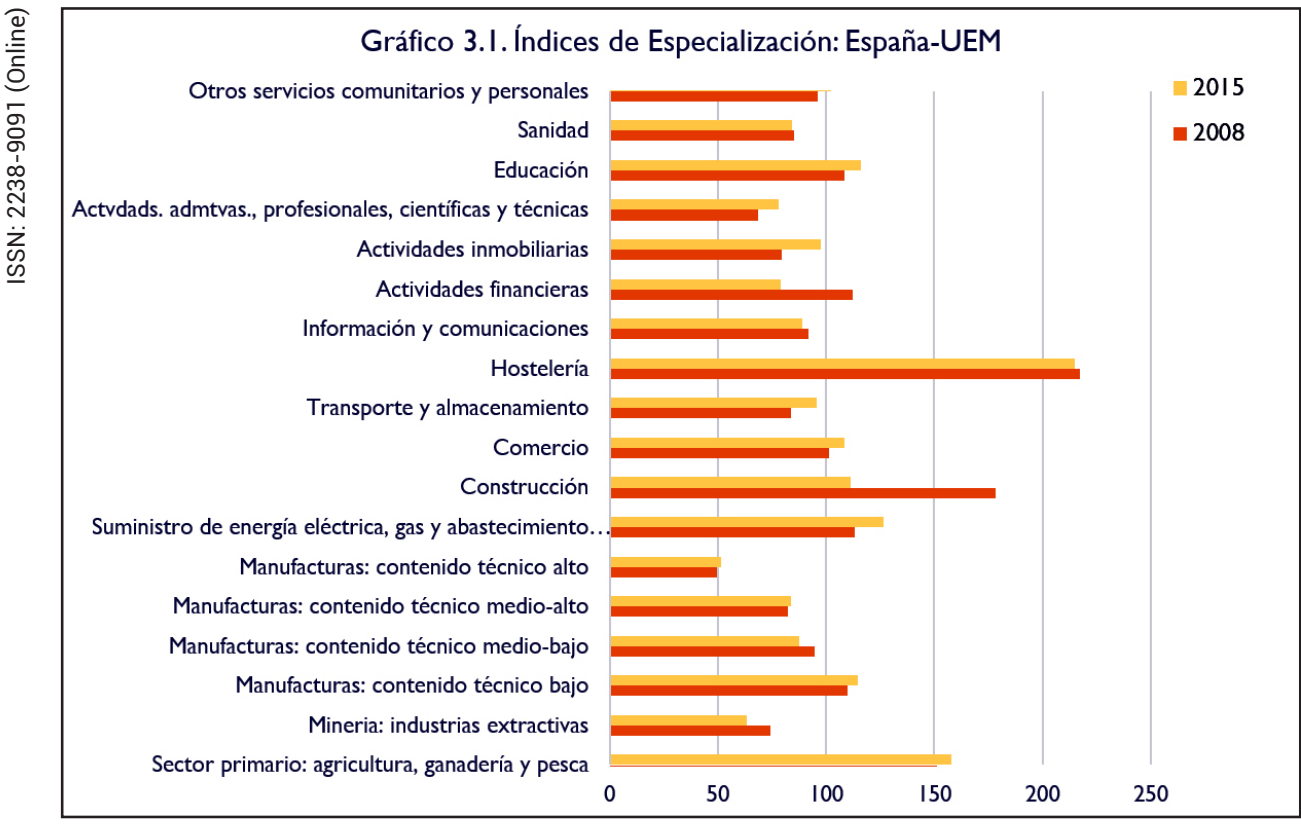

Fuente: elaboración propia a partir de datos de EU Klems.

Las ramas productivas en las que la economía española se encuentra sobreespecializada en relación con el resto de economías europeas en el año 2015 son prácticamente las mismas que en el inicio del periodo: hostelería, sector primario, suministro energético, manufacturas de bajo contenido tecnológico y construcción. Prácticamente todos ellos son sectores productivos poco permeables al cambio técnico. Además, salvo en el caso de la construcción, rama en la que si se ha producido un severo ajuste como consecuencia del estallido de la burbuja especulativa, la sobrerepresentación de estos sectores no se ha corregido a lo largo del periodo. Por el contrario, aquellas ramas productivas más susceptibles a la incorporación del cambio técnico, han permanecido en un segundo plano: el caso de las manufacturas es paradigmático, aunque también destaca la situación del sector de las finanzas así como el de los servicios profesionales, científicos y técnicos.

Esta particular estructura productiva es el principal factor que explica el enjuto avance alcanzado en términos de productividad, que, 
atendiendo a los requerimientos de rentabilidad del capital, ha supuesto una limitación objetiva para el avance de los salarios. De ahí que junto a los mecanismos relativos se hayan activado también los absolutos para potenciar la p', desencadenando así una profunda regresión salarial que late bajo la desigualdad creciente.

\section{2. ¿Cómo se abordan los problemas derivados de las crecientes dificultades de valorización?}

Las tensiones que azotaron a la ganancia cortocircuitaron el proceso de acumulación abriendo un proceso de profunda destrucción socioeconómica. Durante el periodo más agudo de la recesión, entre 2008 y 2013, la formación bruta de capital fijo acumuló una caída de más del 35\% y la tasa de paro se vio incrementada desde el 11,3\% hasta el 26,1\% (Fuente: cálculos propios a partir de AMECO).

La virulenta reacción que adoptó el capital en la economía mundial desde el estallido de la crisis se puede entender como una profundización en la estrategia de desvalorización de la fuerza de trabajo desplegada desde décadas atrás. Concretamente, se puede considerar que la respuesta se erigió en torno a dos ejes interconectados: degradación de las condiciones laborales y contención salarial.

Al quedar mediada la inserción de la economía española a la economía mundial por el proyecto de integración europeo, no es posible comprender la respuesta frente a la crisis en el caso español sin atender a la reacción emprendida desde las instituciones comunitarias. Más si cabe teniendo en cuenta que la Unión Económica y Monetaria (UEM) no contaba con instrumentos específicos para abordar las crisis y se acaba improvisando la institución de un fondo de rescate, el Mecanismo Europeo de Estabilidad (MEDE), en el que participa el propio Fondo Monetario Internacional (FMI). Esta institución dejó su impronta en el diseño de este instrumento: la asistencia financiera ofrecida queda condicionada al cumplimiento de estrictas condiciones en el marco de política económica orientadas a recuperar 
la rentabilidad a costa de la desvalorización de la fuerza de trabajo (ARRIZABALO, 2014). Desde que acude al MEDE se agudiza la subordinación del Estado español frente a los requerimientos de las instituciones comunitarias. Si bien es verdad que la moneda única, desde su puesta en marcha, se había diseñado como un instrumento para sojuzgar los salarios, el estallido de la crisis sirvió para desenmascarar su verdadero rostro (DEL ROSAL y MURILLO, 2015).

Concretamente, la respuesta comunitaria se materializó en dos acuerdos dirigidos a domeñar el avance salarial. Por una parte, el núcleo del Pacto del Euro (2011) lo integra la estrategia de contención salarial sustentada sobre la propuesta de reducir los costes laborales unitarios. De esta manera, las instituciones comunitarias se inmiscuyen en el proceso de fijación de salarios, con el objetivo de subordinar su avance a la mejora competitiva. Para ello se propone que los eventuales progresos de los salarios reales no agoten las mejoras en términos de productividad, garantizando así el mecanismo relativo de extracción de plusvalor, y se promueve la abolición de las cláusulas de salvaguarda así como la descentralización del marco de negociación colectiva. Asimismo, conmina a los Estados miembros a moderar el avance del salario de los empleados públicos, en la misma senda de contención definida para el sector privado.

Por otra parte, el Pacto Fiscal de 2012, integrado en el Tratado de Estabilidad, Coordinación y Gobernanza, supuso la incrustación de la disciplina fiscal en la Constitución española. Mediante la reforma del artículo 135 se subordina la actuación de las Administraciones Públicas al principio de estabilidad presupuestaria, asumiendo el techo de déficit público definido por la UE y concediendo prioridad absoluta al pago de la deuda sobre el resto de las partidas del gasto público. Además, se ha reforzado el mecanismo sancionador vinculado al PEC mediante el Semestre Europeo, constriñendo aún más el marco de política económica, que afecta a dimensiones en las que no hay una cesión plena de soberanía nacional, como es el caso de la política laboral. 
Estas directrices definieron el escenario en el que se han concretado las medidas emprendidas en España para abordar las tensiones sobre la rentabilidad, que situaron en el ojo del huracán el conflicto distributivo. En un intento descarnado por revertir el deterioro de las condiciones de ganancia se profundizó el ajuste salarial sobre el que se había asentado el patrón de crecimiento durante la fase previa al estallido de la crisis. La reacción puede ser entendida como una vía para incrementar la p' mediante el despliegue de una estrategia integral para someter a los salarios a los requerimientos de la rentabilidad. Para ello, se adoptaron medidas para subyugar a los diferentes componentes del salario.

En primer lugar, cabe destacar el impacto que sobre el componente indirecto de los salarios generaron los recortes del gasto público de carácter social (BANDRÉS, 2016). La reacción del Estado frente a la crisis quedó focalizada en el rescate financiero mediante el que estatalizó una fracción importante de la deuda privada de las empresas, acudiendo así al auxilio de ciertos segmentos del capital. Fundamentalmente, esta intervención explica que la deuda privada descendiera desde el $201,4 \%$ de 2009 hasta el 154,6\% de 2015, definida en ambos casos sobre el PIB, mientras que, durante ese mismo periodo, la deuda pública sobre el PIB prácticamente se duplicó (desde el 52,8\% hasta el 99,4\%). Por lo tanto, conviene secuenciar ordenadamente el relato de los hechos para comprender la lógica interna del proceso: la explosión de los niveles de deuda pública no puede entenderse como la causa explicativa de la crisis sino como uno de los resultados de la reacción adoptada frente a ella.

Esto provocó un intenso crecimiento de los intereses, hasta el punto de que se ha convertido en la rúbrica que más recursos absorbe del presupuesto estatal (más allá del gasto en pensiones): más de 35.000 millones de euros en el año $2015^{6}$. Al haberse recogido en la Constitución la prioridad absoluta de devolución de la deuda pública, así como de los intereses derivados de ella, el pago de estos 
compromisos se ha realizado en detrimento de otros componentes del gasto público, fundamentalmente aquellos de carácter social. Los recortes quedaron focalizados en sanidad, enseñanza y dependencia, que representan una parte importante del componente indirecto de los salarios. Así como en los salarios de los empleados públicos. Como se puede comprobar, el crecimiento de los niveles de deuda pública sirve como coartada para aplicar recortes de enorme relevancia en el componente indirecto del salario.

Y todo ello en un contexto en el que se aliviaron las cargas fiscales sobre el capital, renunciando así a una ingente masa de recursos. La reforma fiscal se articuló en torno a tres medidas de profundo carácter regresivo: reducción del tipo impositivo del Impuesto de Sociedades, desde el 30\% hasta el 25\%, apertura de un proceso de amnistía fiscal e incremento del tipo impositivo general del IVA desde el 18\% hasta el 21\%, así como una reducción del conjunto de productos que disfrutan de un tipo reducido.

El proceso de subyugación salarial alcanzó también al componente diferido del salario. En pleno proceso de explosión de la tasa de paro se redujo la cuantía de la prestación contributiva por desempleo: a partir del sexto mes en esta situación el trabajador deja de cobrar el $60 \%$ de la base reguladora para comenzar a recibir un 50\%.

Mención aparte merece las medidas aplicadas sobre el sistema público de pensiones por jubilación, que representan el principal componente del salario diferido. Además de congelar las pensiones en el año 2011, el Gobierno del PSOE implementó una de las contrarreformas más duras a las que se ha sometido al sistema, que pivotó sobre el retraso de la edad de retiro, la ampliación del periodo de cómputo para el cálculo de la base reguladora y el incremento del periodo de cotización para generar el derecho a recibir la prestación plena.

Dos años más tarde el Gobierno del Partido Popular ejerció una nueva vuelta de tuerca sobre el sistema (Ley 23/2013), incrustando dos elementos de claro corte regresivo. Por una parte, la introducción del factor de sostenibilidad para corregir a la baja automáticamente la 
cuantía de la prestación a recibir a medida que aumenta la esperanza de vida. Por otra parte, se rompe con el compromiso legal de mantenimiento del poder adquisitivo, a partir del vínculo del avance de la cuantía de las pensiones con el del IPC, ligándolas a partir de ese momento a un índice de revalorización, definido según la situación financiera de la Seguridad Social y mermando así su capacidad protectora.

Además, se profundizó aún más en la senda de desregulación laboral abierta décadas atrás, adoptando medidas de tal calado que pulverizan el marco de negociación colectiva existente hasta el momento (PÉREZ, 2015). En primer lugar, se invierte la relación jerárquica mantenida por los acuerdos en sus diferentes niveles de negociación, primando los convenios de empresa sobre el resto. Asimismo, se permitió la modificación unilateral por parte de las empresas de las condiciones sustanciales de la relación laboral, alegando únicamente la necesidad de mejorar su competitividad. Incluso se promovió un escenario más laxo para la aplicación de las cláusulas de descuelgue.

También se modificaron tanto las condiciones de entrada como las de salida del mercado laboral a través de diferentes medidas que perseguían el abaratamiento de la fuerza de trabajo. En primer lugar, se favoreció el uso de las ya generalizadas formas precarias de contratación y se implementó un nuevo tipo de contrato que ampliaba el periodo de prueba hasta el año. Asimismo, se abarató el despido generalizando el contrato de fomento de la contratación indefinida y relajando los criterios para acogerse a los despidos por causas objetivas.

La reducción de la tasa de paro que se aprecia en los últimos años no debe de nublar la visión a la hora de analizar la situación del mercado laboral. Hay ciertos colectivos cuya vulnerabilidad se ha extremado. El desempleo de larga duración, que en 2008 afectaba al 18\% del total, en el año 2017 alcanzaba ya a prácticamente la mitad de los parados (44,5\%). Los más jóvenes acusan especialmente el problema del desempleo, que afecta al 38,5\% de los activos, frente al 18,6\% promedio de la eurozona en $2017^{7}$. 
Además, la caída del desempleo no se corresponde únicamente con la creación de empleo, sino que también queda explicada por la emigración, el retorno de inmigrantes y el abandono de la población activa por parte de ciertos colectivos. Asimismo, el empleo generado se ha caracterizado por su elevado grado de precariedad. Especialmente en la forma de contratos parciales, que ya afectan al 16\% del total de ocupados, entre los que abunda el subempleo: en el año 2017 hasta un 57,3\% de los trabajadores contratados bajo esta modalidad, afirmaban haberlo aceptado tras buscar infructuosamente un trabajo a jornada completa ${ }^{8}$. Y la temporalidad sigue cronificada en unos niveles muy elevados (26,8\% del total de ocupados) frente a la media de la eurozona (16\%)

El último de los resortes activados para contener el avance salarial lo representan los Acuerdos Interconfederales de Negociación Colectiva, que ya se habían utilizado durante la fase previa para garantizar la contención salarial. El principal contenido de los dos acuerdos alcanzados durante el periodo lo representa la imposición de techos definidos a nivel estatal para el progreso de los salarios, de marcado corte restrictivo. De este modo, el marco de negociación quedo constreñido lo que permitió completar el marco de regresión salarial implementando una selectiva política de rentas confinada a los salarios.

Todas estas medidas apuntan en el mismo sentido: la recuperación de las condiciones de ganancia para reactivar la acumulación a costa del incremento de p.' El marco de negociación colectiva más descentralizado, junto al establecimiento de techos para el avance salarial, promovió la desvalorización de la fuerza de trabajo. La constricción del componente directo del salario se ha reforzado promoviendo el uso de los contratos precarios, especialmente el de jornada parcial. El componente indirecto del salario también se ha visto comprimido como consecuencia de los recortes sociales del gasto público. Y, por último, el abaratamiento del despido, la reforma de la prestación por desempleo y, especialmente, la contrarreforma aplicada en el sistema público de pensiones, erosionaron el componente diferido del salario. 
Estos han sido los principales elementos constitutivos de la beligerante reacción adoptada por el capital a efectos de recuperar las condiciones de rentabilidad a costa de intensificar las condiciones de explotación de los asalariados. ¿Qué efectos tuvieron en términos de desigualdad?

\section{Impacto de la crisis en términos de desigualdad}

El azote de la crisis sobre las condiciones materiales de vida ha sido severo: solo en el año 2017 se ha conseguido recuperar el nivel de ingresos per cápita del año 2007. Además, ha tenido un notable impacto sobre la desigualdad: en la Tabla 4.1 se recogen varios indicadores que lo constatan. La ratio que relaciona el ingreso del 20\% de población más rico y el del 20\% más pobre sufrió un crecimiento del $17,8 \%$, mostrando así un ensanchamiento de las diferencias muy superior al promedio de la eurozona, donde prácticamente se mantuvieron. La trayectoria del índice de Gini también refleja el crecimiento de las diferencias en la distribución del ingreso: mientras que en la eurozona se mantuvo prácticamente constante, en el caso español ascendió significativamente, situándose solo por detrás de los niveles de Lituania y Letonia.

Tabla 4.1. Distribución personal del ingreso

\begin{tabular}{|l|c|c|c|c|}
\hline \multirow{2}{*}{} & \multicolumn{2}{|c|}{ España } & \multicolumn{2}{c|}{ Eurozona } \\
\cline { 2 - 5 } & 2008 & 2017 & 2008 & 2016 \\
\hline Coeficiente 80/20 & 5,6 & 6,6 & 4,9 & 5 \\
\hline Índice de Gini & 32,4 & 34,1 & 30,5 & 30,4 \\
\hline
\end{tabular}

Fuente: elaboración propia a partir de datos de Eurostat

Integrando en el análisis la distribución del patrimonio familiar se puede detectar una tendencia similar durante el último periodo, si bien es verdad que en este caso las cotas de desigualdad alcanzadas son más elevadas: el Índice de Gini en riqueza neta crece desde el 0,6 has- 
ta el 0,7 desde 2008 hasta 2014 (Fuente: Encuesta Financiera de las Familias (EFF), Banco de España (BE)). En la Tabla 4.2 se puede comprobar cómo el segmento de población más rico acaparó una fracción creciente de la riqueza neta a lo largo del periodo.

Tabla 4.2. Distribución de la Riqueza neta

\begin{tabular}{|l|c|c|c|}
\hline & $\begin{array}{c}1 \% \text { CON MAYOR } \\
\text { RIQUEZA }\end{array}$ & $\begin{array}{c}\text { 5 \% CON MAYOR } \\
\text { RIQUEZA }\end{array}$ & $\begin{array}{c}10 \% \text { CON MAYOR } \\
\text { RIQUEZA }\end{array}$ \\
\hline $\mathbf{2 0 0 8}$ & $\mathbf{1 5 , 3}$ & 31,7 & 44,4 \\
\hline $\mathbf{2 0 1 1}$ & $\mathbf{1 6 , 9}$ & 34,2 & 46,5 \\
\hline 2014 & 20,2 & 40,4 & 52,8 \\
\hline
\end{tabular}

Fuente: $\mathrm{EFF}, \mathrm{BE}$.

Solo es posible comprender las causas de la creciente desigualdad manteniendo una perspectiva de clase. El creciente antagonismo entre los intereses de propietarios y asalariados se agudiza en las fases recesivas. El capital ha intentado aliviar la presión creciente sobre la rentabilidad mediante una estrategia que ha supuesto un ataque directo a los salarios por lo que para entender el escenario de desigualdad creciente se debe escudriñar la pugna distributiva básica entre capital y trabajo.

En primer lugar, cabe destacar que los salarios reales han quedado prácticamente estancados: durante el periodo 2009-2017 la tasa media anual acumulativa (TMAA) del salario medio por hora de trabajo, una vez descontado el efecto de la variación de precios, ha sido del 0,24\% ${ }^{10}$.

En trabajos similares - Murillo (2019a) y Buendía, Molero y Murillo (2018) - se ha descartado que el repunte de la desigualdad responda a una mayor dispersión salarial. Por lo tanto, para comprender las raíces de la creciente desigualdad se hace necesario poner de manifiesto la trayectoria conjunta de beneficios y salarios. Para ello, en el Gráfico 4.1 se representa la trayectoria del salario relativo (SR) y del beneficio relativo (BR). El primero de estos indicadores refleja el peso relativo de la masa salarial, aproximada a través de la remuneración de asalariados, sobre la renta total, según se ha detallado en la ex- 
presión 2.1. El beneficio relativo se obtiene como la relación entre el conjunto de rentas de la propiedad sobre la renta total"1.

\section{Gráfico 4.1. Distribución relativa del ingreso}

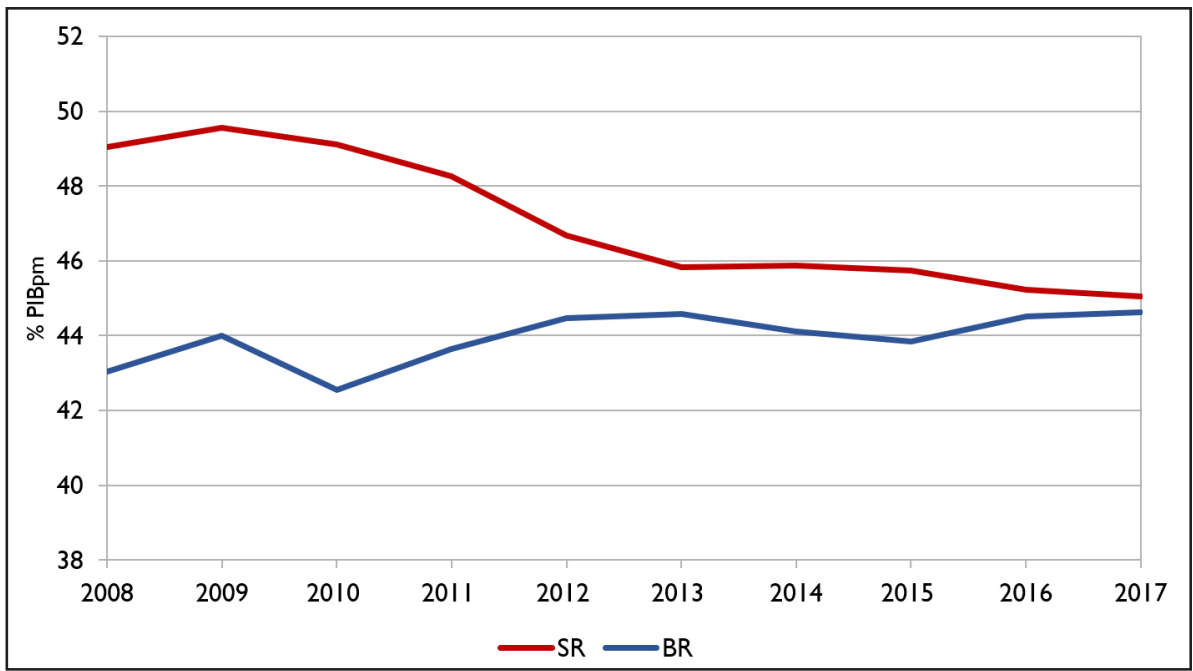

Fuente: elaboración propia a partir de INE y EPA.

El análisis del Gráfico 4.1 permite detectar tendencias de carácter opuesto para cada uno de los dos indicadores. Mientras que los beneficios y el resto de las rentas de la propiedad absorbieron una fracción creciente del ingreso total, mejorando su posición en un 3,6\%, el SR quedó sometido a una involución significativa, perdiendo más de un 8\% de la porción del ingreso que acaparaba en el año 2008.

No obstante, la profundidad de la regresión salarial no puede ser captada sin atender a la evolución de la estructura social: una vez que se ha valorado cómo se distribuyó el ingreso entre los distintos tipos de renta, resta integrar el análisis de los sujetos que se apropiaron de ellas.

Guerrero (2006) propone el cálculo del coeficiente salarial para integrar ambas tendencias en un único indicador que se obtiene relacionando el SR y la tasa de asalarización. De manera análoga, relacionando el BR con el peso relativo de los propietarios sobre la población 
total, se puede calcular el coeficiente de beneficios. En la Tabla 4.3 se muestra el valor de ambos coeficientes durante el periodo analizado. Para reflejar una situación de equidistribución entre clases sociales, el indicador debería tomar el valor de 1; cuanto más se acerque el valor del indicador a 0 peor situada estará la clase social analizada en el marco de relaciones de distribución del ingreso.

\section{Tabla 4.3. Coeficiente salarial y de beneficios}

\begin{tabular}{|l|c|c|}
\hline & 2008 & 2017 \\
\hline Coeficiente salarial & 0,61 & 0,58 \\
\hline Coeficiente de beneficios & 8,47 & 10,5 \\
\hline
\end{tabular}

Fuente: elaboración propia a partir de datos del INE y de EPA.

Como se puede observar, el coeficiente salarial experimentó una caída del 4,9\%, reflejando así de manera más precisa la involución a la que quedaron sometidos los salarios durante el periodo, mientras que los propietarios reforzaron su dominio en la distribución del ingreso. En este creciente antagonismo entre capital y trabajo radican las causas de la creciente desigualdad observada en la economía española desde el estallido de la crisis.

\section{Conclusiones}

La creciente desigualdad observada en la economía española durante el periodo reciente no puede ser entendida más que desde una perspectiva de clase. El antagonismo existente entre el capital y el trabajo se manifiesta de manera más cruda a partir del estallido de la crisis, cuando se activan una serie de resortes para garantizar una distribución del ingreso favorable a los beneficios. Aunque esta reacción ha mostrado cierta línea de continuidad con el patrón mantenido durante la fase previa, pivotando sobre la contención salarial y la degradación de las condiciones laborales, la profundidad de las reformas y la beligerancia con la que se han implementado sitúan a la 
reacción emprendida para aliviar la presión sobre la rentabilidad en un plano cualitativamente distinto.

El impacto de la crisis sobre el mercado laboral, así como los mecanismos desplegados para insuflar un grado adicional de precariedad que fuera funcional para desvalorizar la fuerza de trabajo, han dilatado el ejército industrial de reserva durante los últimos. No solo en la forma de desempleados, sino que también han asumido un protagonismo creciente los subempleados, los temporales y los trabajadores irregulares, generando así una presión adicional sobre la dinámica salarial. El ajuste desplegado sobre los salarios ha tenido un carácter integral, afectando así a sus diferentes componentes (directo, indirecto y diferido). Sin embargo, la recesión no ha servido para modificar sustancialmente la estructura productiva, más allá del retroceso experimentado por el sector de la construcción. De ahí que el enjuto avance de la productividad haya supuesto una limitación objetiva para el avance de los salarios, bajo la lógica de acumulación capitalista.

El método de análisis marxista ofrece las herramientas necesarias para comprender que la creciente desigualdad no responde a fenómenos únicamente coyunturales. Y permite comprender que no puede resolverse exclusivamente en el plano distributivo ya que las dificultades de generación del plusvalor representan la principal contradicción a la que se enfrenta el capital de manera inexorable y como resultado de su lógica ordinaria de funcionamiento. El capital mantiene la necesidad de explotar crecientemente a los trabajadores, por lo que la lucha contra la desigualdad exige la superación de la acumulación basada en la lógica de la rentabilidad.

\section{Bibliografía}

ARRIZABALO, Xabier. Capitalismo y economía mundial. Madrid, IME, 2014.

BANDRÉS, Eduardo: “Gasto social, redistribución y desigualdad”, Papeles de economía española, España, n 147, p. 128-144, 2016. 
BOUNDI, Fahd: "Tasa de beneficio y distribución del ingreso en la economía española", Ensayos de Economía, Colombia, n 44, p. 75-99, 2014.

BUENDÍA, Luís; MOLERO, Ricardo y MURILLO, F. Javier (2018): "The distributive pattern of the Spanish economy: the impact of adjustment on inequalities", en BUENDÍA, Luís y MOLERO, Ricardo (ed.), The political economy of contemporary Spain. From Miracle to Mirage. Abingdon, Routledge, p. 124-149.

DE ARRIBA, Raúl: “Crisis, política económica y desigualdad en España”, Papeles de Europa, España, vol. 27, p. 70-84, 2014.

DEL ROSAL, Mario y MURILLO, F. Javier. "Acumulación y crisis en la zona euro", en MATEO, Juan Pablo (coord.), Capitalismo en recesión. La crisis en el centro y la periferia de la economía mundial, Madrid, Maia Ediciones, p. 137-175, 2015.

GILL, Louis. Fundamentos y límites del capitalismo. Madrid, Trotta, 2002.

GUERRERO, Diego. La explotación. Trabajo y capital en España (1954-2001). Barcelona, El Viejo Topo, 2006.

MARX, KARL. Salario, precio y ganancia. Fundación Federico Engels, Madrid, 2003 [1865].

MATEO, Juan Pablo: "Las causas de las crisis económicas en España", Filosofía, política y economía en el Laberinto, España, n 42, p. 83-96, 2014.

MURILLO, F. Javier. Crisis, explotación y salarios: análisis marxista del caso español, Revista Análisis Económico, México, Universidad Autónoma Metropolitana, vol. XXXIV, nº 87, p. 101-124, 2019a.

MURILLO, F. Javier. El milagro económico español. Dinámica salarial e impacto sobre la estructura de propiedad, Maia, Madrid, 2019b.

PÉREZ, J. Ignacio: "Las reformas laborales en la crisis económica: su impacto económico”, Ekonomiaz, España, n 87, primer semestre, p. 247-280, 2015.

ROSER, Max y CRESPO, Jesús: "Why is income inequality increasing in the developed world?", Review of income and wealth, series 62, n 1, 2016.

\section{NOTAS}

1 Profesor Asociado de la Universidad Complutense de Madrid. España. ORCID number: 0000-0001-9663-1226. fjmurill@ucm.es

2 Para una explicación detallada sobre la teoría del salario de Marx véase el capítulo 1 de Murillo (2019b). 
3 Para una explicación metodológica sobre el cálculo de la tasa de ganancia véase Murillo (2019b).

4 En este caso se ha optado por utilizar el valor añadido para el cálculo de los pesos relativos en cada uno de los casos analizados. Los subsectores industriales se han agrupado según su grado de intensidad tecnológica de la siguientes manera:

- Contenido tecnológico alto: industria farmacéutica, maquinaria informática y de oficina, material de comunicación e instrumental médico y óptico.

- Contenido tecnológico medio-alto: química y productos químicos (salvo industria farmacéutica), resto maquinaria, maquinaria y equipo eléctrico, vehículos de motor y otro equipamiento de transporte

- Contenido tecnológico medio-bajo: refinado de petróleo y coque, plástico y caucho, otros minerales no metálicos, metales básicos y metales fabricados.

- Contenido tecnológico bajo: alimentos y bebidas, tabaco, textil, preparación y teñidos de pieles, cuero y calzado, madera y corcho, papel, resto de manufacturas y reciclaje.

5 Fuente: Comisión Europea. Anexo estadístico del Informe sobre el Mecanismo de Alerta 2018.

6 Fuente: Ministerio de Hacienda y Administraciones Públicas

7 Todos los datos del párrafo proceden de Eurostat.

8 Todos los datos del párrafo proceden de la EPA, INE.

9 Fuente: Eurostat.

10 Cálculos propios a partir de los datos del INE. Para corregir el efecto de la inflación se ha utilizado el deflactor del PIB.

11 La masa salarial se ha aproximado a través de la remuneración total de asalariados. Para el conjunto de rentas derivadas de la propiedad se ha usado una versión ajustada del Excedente Bruto de Explotación que ofrece el INE. De este agregado se ha descontado el ingreso de los trabajadores autónomos. Al no estar disponible el dato de la renta de los autónomos se ha estimado considerando que su ingreso fue equivalente al salario medio durante el periodo. 


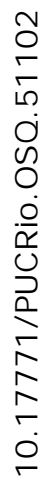

용 